\title{
LA SOSTENIBILIDAD FISCAL Y LA REGRESIVIDAD DE DERECHOS SOCIALES: UN PRIMER MOMENTO DE ANÁLISIS
}

Natalia Juliana Pacheco Quintero María Alejandra Jaimes Sanguino Annie Stefane Duarte Bernal ${ }^{1}$

Recibido: Enero 20 de 2017 Aprobado: Mayo 7 de 2017

\section{RESUMEN}

A partir del análisis de los debates de aprobación del Acto Legislativo 03 de 2011 y de la Sentencia C-288 del 2012, primer momento de existencia de la sostenibilidad fiscal, se encuentra con que aquella y el incidente de impacto fiscal se incorporan como instrumentos para la protección y garantía de los derechos sociales. De tal suerte, permite su enlace con los rasgos del Juez neoconstitucional-garantista de derechos sociales, autoconstruidos por la Corte Constitucional a partir de la cláusula de Estado social de Derecho y las tesis de justiciabilidad de los derechos sociales.

Palabras clave: Derechos sociales, sostenibilidad fiscal, Corte Constitucional, Principio de progresividad.

\section{FISCAL SUSTAINABILITY AND RETROGRESSIVE MEASURES IN SOCIAL RIGHTS: A FIRST MOMENT OF ANALYSIS}

\begin{abstract}
Based on the analysis of the debates of approval of Legislative Act 03 2011 and Decision C-288 of 2012, which are the starting point of fiscal 1 Estudiantes del Programa de Derecho de la Universidad Autónoma de Bucaramanga. Integrantes del Semillero de Investigación en Hermenéutica Jurídica -HERMES- adscrito al Grupo de investigación en Hermenéutica Jurídica del Centro de Investigaciones Socio-Jurídicas "Laureano Gómez Serrano". hermes@unab.edu.co
\end{abstract}


sustainability, it was found that the latter and the incidental plea of fiscal impact are considered as instruments for the protection and guarantee of social rights. In that way, it is possible to connect them with the features of the neoconstitucional Judge, guarantor of social rights, which have been self-constructed by the Constitutional Court based on the social State of law clause and the thesis of the justiciability of social rights.

Key Words: Social rights, fiscal sustainability, Constitutional Court, the principle of progressivity.

\section{A SUSTENTABILIDADE FISCAL E REGRESSIVIDADE DE DIREITOS SOCIAIS: UM PRIMEIRO MOMENTO DE ANALISE RESUMO}

A partir da análise dos debates de aprovação do Ato Legislativo 03 de 2011 e da Sentença C-288 de 2012, o primeiro momento da existência da sustentabilidade fiscal, se encontra com que aquela e o incidente de impacto fiscal são incorporados como instrumentos para a proteção e garantia dos direitos sociais. Dessa forma, ele permite sua ligação com as características de Juiz neoconstitutional-garantista de direitos sociais, auto-construídos pelo Tribunal Constitucional a partir da cláusula do Estado social de Direito e as teses da justiciabilidade dos direitos sociais.

Palavras-chave: Direitos sociais, sustentabilidade fiscal, Tribunal Constitucional, princípio da progressividade.

\section{INTRODUCCIÓN}

A pesar de que expresamente la Constitución de 1991 no prevé una acción constitucional para garantizar la eficacia de los derechos sociales, como sí lo hace con los derechos fundamentales y los derechos colectivos, la jurisprudencia de la Corte Constitucional vía interpretación los ha hecho posible al considerar que también son derechos fundamentales. La garantía de los derechos sociales exige tanto obligaciones de abstención como de prestación, las cuales demandan las 
más de las veces la erogación de recursos públicos, toda vez que a través de ellos se entregan bienes escasos y caros.

La declaratoria de estados de inconstitucionalidad y en general las sentencias estructurales, referidas, por ejemplo, a los derechos de las personas privadas de la libertad en las cárceles, los de los desplazados, y los usuarios del sistema de la salud, altera los presupuestos promovidos por los Gobiernos Nacionales. Ferrajoli (2011) ha dicho que son los derechos sociales el espacio de lo que no se puede dejar de decidir en las democracias actuales, por lo que estas sentencias judiciales han sido recibidas por cierta parte de la doctrina como un cierre a las brechas que genera los espacios de decisión representativa en los que no se ven reflejados "los últimos de la fila".

Sin embargo, en otro sector, tal vez minoritario, y en los Gobiernos Nacionales, en general, tales órdenes son vistas como una extralimitación de la competencia de la Corte Constitucional, y un desquiciamiento de las finanzas públicas que impiden una adecuada planeación del cumplimiento de los fines esenciales del Estado.

Estas discusiones se enmarcan en un debate global que viene de tiempo atrás, sobre la legitimidad de los jueces para tomar decisiones con impacto económico, en la que Roa (2015) ha identificado cuatro tipo de jueces según la tradición teórica en que se inspire: (i) el Juez liberal restrictivo, (ii) el Juez del constitucionalismo débil, (iii) el Juez del constitucionalismo deliberativo, (iv) el Juez del neoconstitucionalismo y del garantismo ferrajoliano.

En medio de esta tensión, entra en vigencia el Acto Legislativo 03 de 2011, que ordena atender el criterio de "sostenibilidad fiscal" a todas "las Ramas y Órganos del Poder Público" y consagra el "incidente de impacto fiscal" como etapa judicial que puede llegar a surtirse ante "las máximas corporaciones judiciales" en procesos cuyas sentencias pueda tener "consecuencias... en las finanzas públicas"(art.1).

Esta reforma constitucional ha generado temores, pues, como se verá más adelante, se denuncia que puede convertirse en una limitación al trabajo que la Corte Constitucional ha desarrollado para hacer realidad las promesas de la cláusula de Estado social de Derecho presente en la Constitución de 1991, y de ser un retroceso de los derechos sociales.

2 Bajo esta denominación, la Corte Constitucional se ha referido al conjunto de personas que sin la decidida actuación del Estado no tendrían acceso a los derechos sociales: Ver: T-418 de 2010. 
Dentro del Semillero de Investigación en Hermenéutica Jurídica -HERMES - se desarrolla el proyecto titulado "La regresividad de los derechos sociales a partir de la sostenibilidad fiscal en la jurisprudencia de la Corte Constitucional en Colombia (2012-2016)", cuyo objetivo principal es identificar si la Corte Constitucional ha promovido la regresividad de los derechos sociales con fundamento en la reforma constitucional de 2011.

Este escrito presenta un primer avance y resultado parcial de la investigación, en el que se hace un análisis

El interrogante que guía este trabajo y al que se quiere dar respuesta es: ¿si en esta primera comprensión se admite que sea posible utilizar argumentos de sostenibilidad para dar pasos hacia atrás frente garantías ya reconocidas de derechos sociales?

Se adelanta la idea de que tanto en el Congreso de la República como en la Sentencia C-288 de 2012, la sostenibilidad fiscal y el incidente de impacto fiscal se enfocan como instrumentos para el cumplimiento de la cláusula del Estado Social de Derecho, a fin de obtener un equilibrio entre la protección de los derechos fundamentales y el buen manejo del presupuesto estatal; subordinado al principio de progresividad de los derechos sociales y a la prohibición de regresividad.

En escritos futuros, se analizarán casos particulares de derechos sociales en los que se llegue a adoptar decisiones que sean regresivas.

Para los fines de este escrito, metodológicamente se divide en cinco partes: en la primera se presentarán los cuatro modelos o tipos de jueces en relación a la toma de decisiones con impacto económico, como son la mayoría de las sentencias sobre derechos sociales; luego se identificará el modelo que ha venido desarrollando la jurisprudencia de la Corte Constitucional hasta la entrada en vigencia del Acto Legislativo 03 de 2011. Enseguida - partes terceras y cuartas-se dará respuesta al problema atrás planteado, para finalmente exponer unas conclusiones.

\section{LOS MODELOS DE JUEZ FRENTE A LA DECISIONES CON IMPACTO ECONÓMICO}

El profesor Roa (2015) señala que los debates que se han suscitado alrededor del Acto legislativo 03 de 2011, en referencia al incidente de impacto fiscal en él implanto, son muestra de la preocupación de larga 
data que causa el impacto económico de algunas decisiones judiciales, no sólo en Colombia sino en diferentes Estado del mundo.

En su trabajo este profesor externadista aborda las respuestas dadas por la teoría general del Derecho constitucional frente a la cuestión de las "sentencias que cuestan y la legitimidad de los jueces para proferirlas". El propone cuáles son:

\subsection{El juez liberal restringido}

Este Juez tiene como referente teórico al constitucionalismo liberal del siglo XIX, y se edifica con una prohibición de intervenir en la economía. Según esta tradición los jueces no tienen legitimidad democrática para proferir decisiones que impliquen costos o que tengan intervención en la economía. Es así debido a la máxima "dejar hacer, dejar pasar", que excluye al Estado del manejo de la dinámica de la economía. De modo rotundo, se concluye que los jueces no tienen la competencia para proferir sentencias que tengan impacto económico. De tal modo, la protección judicial de los derechos sociales no sería posible.

\subsection{El Juez del constitucionalismo débil}

Se caracteriza por la prevalencia de las respuestas tecnocráticas sobre sus decisiones. Este referente teórico acepta que los jueces tienen facultad para proferir decisiones con consecuencias económicas, pero supone que pueden llegar a ser excesivamente generosas, sin cálculo de sus consecuencias. Así, los sistemas de constitucionalismo débil tienen mecanismos que posibilitan al legislador o al ejecutivo atender sentencias que pueden afectar gravemente la economía del Estado. Son ellos quienes tienen "la última palabra" en lo referente a la protección de los derechos fundamentales, como, ya se verá, son los derechos sociales.

\subsection{EI Juez del constitucionalismo deliberativo}

Se caracteriza por permitir espacios de discusión pública dentro de los procesos judiciales en el que se van a tomar decisiones con impacto económico. Sí reconoce a los jueces como competentes para la adopción de providencias con impacto económico, pero pone de presente que ellos no tienen la capacidad técnica que una democracia exige para tomar decisiones que incluyan aspectos económicos. Este modelo propone mecanismos para asegurarse de que el grado de sensibilidad por efectos económicos se concrete durante el proceso judicial y en la decisión final; 
en virtud de los cuales se consultan a expertos en la materia que ilustran a los magistrados.

\subsection{El Juez del neoconstitucionalismo y del garantismo constitucional}

Inspirado en Alexy y Ferrajoli, este Juez se edifica bajo la convicción de "la autosuficiencia de los métodos de interpretación para racionalizar las decisiones judiciales". Ambos modelos reconocen que el poder judicial: (i) tiene un papel primordial en la garantiza los derechos constitucionales, por lo que (ii) debe adoptar las medidas necesarias para cumplir con dicha función, inclusive aquellas que generen impacto económico.

De cierta manera, a estos modelos resultan irrelevantes los efectos económicos de las decisiones judiciales, pues su racionalidad jurídica se logra en la medida en que en un plano de economía escasa

(...) el juez sabe que si garantiza un derecho (d1) en un grado excesivamente alto, en perjuicio del derecho con el cual éste se encontraba en colisión (d2), esa decisión se traducirá en la disposición excesiva de recursos para garantizar el primer derecho ( $\mathrm{r} 1$ ), en contra de los recursos que se deben disponer para garantizar el derecho que cedió en el juicio de ponderación (r2). (Roa, 2015, p. 10)

Especialmente el garantismo, promueve al principio de progresividad como mecanismo de racionalidad económica. En conclusión, los mecanismos para calcular los efectos económicos de una decisión judicial solo son importantes para quienes deben hacer efectivas las sentencias, para quienes las estudian o para quienes las valoran desde un punto de vista económico.

\section{LA CORTE CONSTITUCIONAL Y LA JUSTICIBIALIDAD DE LOS DERECHOS SOCIALES: UN MODELO JURISPRUDENCIALMENTE CONSTRUIDO}

La posibilidad de que en ejercicio del control de constitucionalidad una alta Corte pueda proferir decisiones amplias que protejan los derechos sociales surge en 1991. La afirmación de Colombia como un Estado social de Derecho, abrió el camino para realizar un Derecho comprometido con la igualdad material, a partir del cual la Corte Constitucional ha diseñado a través de su jurisprudencia un juez robusto 64 
para hacer frente a las amenazas a los derechos sociales, inclusive mediante decisiones con impacto económico.

Esto ha sido posible gracias a los siguientes desarrollos teóricos y conceptuales:

\subsection{Las tesis de Estado social de Derecho que fundamentan al Juez protector de derechos sociales}

Upegui (2009) en una valiosa síntesis de la jurisprudencia constitucional, propone doce tesis de lo que se ha entendido por Estado social de Derecho, de las cuales rescatamos las siguientes:

- La tesis del tránsito: La Constitución de 1991 marca el paso de un Estado Liberal a un Estado Social, en el que el Estado interviene en la economía, se consagran derechos sociales, y la efectividad de los derechos fundamentales es de relevancia jurídica. Esta tesis excluye la posibilidad de que la Corte Constitucional pueda actuar como un Juez liberal restringido,

- La tesis de la reinterpretación de algunos conceptos: Los derechos clásicos se reinterpretan con un nuevo contenido axiológico. Se deben adoptar medidas de discriminación positiva, lo que marca una conexión conceptual entre igualdad real y los derechos sociales. Las sentencias con impacto económico para satisfacer los derechos sociales proferidas por la Corte Constitucional, se edifican en dicha conexión,

- La tesis de inexistencia de poderes ilimitados: Los poderes públicos y privados están sujetos a límites, controles y restricciones. El ejercicio del poder supone ciertos límites en su ejercicio para garantizar su coherencia con el Estado social de Derecho. Las decisiones sobre derechos sociales de la Corte Constitucional en materia de derechos sociales, son un control a actuares omisivos de las autoridades encargadas de satisfacerlos,

- La tesis del compromiso del mínimo vital: Este es un factor determinante del Estado colombiano para los habitantes que son afectados por la pobreza material y su exclusión del Estado. El derecho al mínimo vital se refiere al mínimo básico en salud, educación, alimentación, vivienda y seguridad social, todos vinculados con la dignidad humana,

- La tesis de progresividad de los derechos económicos y sociales: El Estado debe avanzar a partir de mínimos para dar plena satisfacción a los derechos sociales. Para lo cual debe: (i) diseñar políticas públicas, (ii) avanzar cuantitativa y cualitativamente en los 
márgenes de protección de estos, y (iii) no implementar medidas que disminuyan el cumplimiento de los objetivos trazados (principio de no regresividad).

Las exigencias del principio de regresividad se transgrede en dos eventos: (i) cuando se "limite, restringa o reduzca la extensión o el sentido de un derecho social", (ii) o cuando se "imponga a su ejercicio condiciones que con anterioridad no debían sortearse" (Abramovich y Courtis, 2004, p. 122).

\subsection{La tesis de justiciabilidad de derechos sociales}

Ya en otras oportunidades (Duarte, Frías y Ríos, 2014; Ríos 2015), el Semillero HERMES ha recogido el importante labor desarrollada por los profesores javerianos López, Bateman, Vengoechea y López (2009) que identificaron cuatro diferentes tesis de justiciabilidad de los derechos sociales a través de la acción de tutela, en la jurisprudencia de la Corte Constitucional. Estas son:

- La tutelabilidad de derechos sociales que por conexidad deriven en la violación de derechos fundamentales per se,

- La existencia de derechos sociales fundamentales en cabeza de sujetos de especial protección constitucional,

- La transmutación de los derechos sociales de su faceta prestacional a derechos subjetivos con la adopción de medidas legislativas y administrativas de desarrollo,

- La de la "tutelabilidad propia", que derriba la distinción derechos fundamentales/derechos sociales al considerar que todos los derechos de la Constitución, en cuanto a que se relacionen con la dignidad humana, tienen el carácter de derechos fundamentales.

La dinámica judicial lleva a que se vaya decantando caso a caso las exigencias que se derivan de cada derecho social a favor de las personas, y en la medida que los pronunciamientos de la Corte Constitucional adquieren la condición de precedentes judiciales, los derechos sociales pueden ser protegidos hasta por los jueces con reducida competencia legal.

\subsection{Las sentencias estructurales}

Surgen cuando empiezan a darse una serie de casos individuales -sede de tutela-que demuestran que la violación a los derechos fundamentales no es esporádica u ocasional sino generalizada. Esta situación se deriva 66 
"de fallas sistemáticas en la administración pública", por lo que las soluciones a esos casos no contribuyen a solucionarlas sistémicas. Para ello hace falta, además de órdenes concretas, órdenes para la adopción de medidas generales al aparato estatal que vinculan a muchas autoridades.

Esas órdenes se encargan de especificar objetivos, señalar políticas públicas a desarrollar, e identificar medios adecuados de cumplimiento. "[A]lgunos mandatos estructurales son demasiado costosos y dan lugar a que se formulen las objeciones basadas en el costo de la protección" de los derechos sociales (Tushnet, 2013, pp. 134-135).

\subsection{Audiencias y autos de seguimiento}

Como el cumplimiento de las sentencias estructurales no se agota en un solo acto, la Corte Constitucional con fundamento en el artículo 25-2 lit. c) de la Convención Americana de Derechos Humanos (1969)3 creado jurisprudencialmente las audiencias de seguimiento en donde la Sala que profirió la sentencia estructural cita a las autoridades públicas vinculadas a ellas a fin de que expongan las medidas de cumplimiento adoptadas y su éxito, escuchando a la comunidad y personas interesadas en la garantía de los derechos fundamentales.

Según lo recaudado o debatido en estas sentencias, la Corte Constitucional llega a producir autos de seguimiento en donde van modificando sus decisiones -reconociendo avanzas o intensificando las ordenes en determinados sectores-para lograr la eficacia de la sentencia estructural ${ }^{4}$.

Este diseño jurisprudencialmente creado demuestra que la Corte se reconoce a sí misma como un juez del neoconstitucionalismo y garantismo constitucional, el cual tiene un alto nivel de legitimidad democrática, es decir, que determina que el poder judicial tenga una función garante de los derechos constitucionales y, por esto, adopta las medidas requeridas para cumplir con dicha función, de modo que los efectos económicos se tienen en cuenta cuando se efectivizan las sentencias, cuando se estudian y cuando se valoran desde el punto de vista económico. Dicho esto, se entiende que la protección de los derechos constitucionales debe ser garantizada en todo momento y los recursos que se empleen para este fin no pueden ser puestos como elemento primordial dentro de la jurisprudencia.

3 Los Estados se obligan "a garantizar el cumplimiento... de toda decisión en que se haya estimado procedente el recurso"

4 Desde luego no a toda sentencia estructural le sigue audiencias y autos de seguimiento. Casos ejemplarizantes en que sí, son las de las Sentencia T-024 de 2005 y T-760 de 2008. 


\section{ANÁLISIS DE LOS DEBATES EN EL CONGRESO DE LA REPÚBLICA PARA LA APROBACIÓN DEL ACTO LEGISLATIVO 03 DE 2011}

En el proyecto de acto legislativo 03 (2011) presentado por el Gobierno Nacional, se presenta a la sostenibilidad fiscal como un derecho que se enmarca en los objetivos de racionalizar la economía para mejorar la calidad de vida de los colombianos, promover la distribución equitativa de oportunidades y beneficios del desarrollo, así como tener un ambiente sano; todo lo anterior con el fin de lograr los objetivos del Estado Social de Derecho, teniendo como prioridad el gasto público. El proyecto se interesa por llegar a emplear recursos humanos y asegurar el acceso efectivo al conjunto de bienes y servicios básicos, para aquellas personas que tengan menores ingresos.

Sin embargo, pese a esta visión optimista de la compatibilidad del proyecto de reforma con la Constitución, dentro del Congreso de la República se hicieron intensos debates sobre la afectación al Estado Social de Derecho y a la independencia de la Corte Constitucional.

Se puede agrupar en dos categorías las posturas asumidas por los congresistas, presentándose varias matices dentro de ellas, las cuales son:

\subsection{La sostenibilidad fiscal afecta los derechos sociales}

Estas posturas señalan que al configurarse la sostenibilidad fiscal como principio, puede restringir los derechos fundamentales.

El representante Alfonso Prada cuestiona desde el primer debate si la Constitución colombiana tiene lo necesario para la implementación de la sostenibilidad fiscal o si es necesaria la incorporación de más instrumentos para la misma. Crítica que consagrar a la sostenibilidad fiscal como un principio constitucional simbolizaría el privilegio de la economía por encima de los derechos fundamentales, atentando así contra el Estado Social de Derecho presente en Colombia. Esta postura la sostuvo a lo largo de los debates, denunciando que al verse la sostenibilidad fiscal como un mandato de optimización y por tener la categoría de principio, se sobrepone automáticamente al derecho a la vida, el derecho a la salud, el derecho a la educación o el derecho a la

5 Para la reconstrucción de los debates, el grupo se guio por las referencias presentes en la Sentencia C-1052 de 2012 en el que de manera completa y sucinta se compilan las diferentes posturas a lo largo del trámite de aprobación. 
vivienda digna entre otros derechos. Por esa razón, concluye, es tremendamente equivocado elevarla a categoría de derecho.

Siendo coincidente con el anterior, la representante Adriana Franco dejó en claro que la sostenibilidad fiscal no podía constituirse en el argumento para que el Gobierno Nacional empezara a limitar los derechos y a evitar cumplir con los fines sociales del Estado.

El Representante Alfredo Bocanegra, fue más crítico al manifestar que el proyecto de acto legislativo se propone más que reformar el artículo 334 de la Constitución de 1991, pretende modificar su Preámbulo. Resalta que con la propuesta del Gobierno se busca que la Constitución garantice los derechos del Estado, lo que es absurdo pues los titulares de los derechos son los ciudadanos. Recuerda que si se pone en una balanza la fortaleza y dominancia del Estado y la del ciudadano individualmente considerado, siempre saldrá victorioso el Estado. Insiste en los derechos hay que enfocarlos en virtud del ciudadano, que en un caso determinado no tiene cómo defenderse de los abusos del Estado.

De otra parte, el Representante Carlos Edward Osorio Aguilar adujo que la sostenibilidad fiscal ya está garantizada a través del Estatuto Orgánico del Presupuesto, por lo que la reforma constitucional propuesta es innecesaria. Pero advierte que si lo que con ella se busca es elevarla a canon constitucional, es para enfrentarla con los derechos fundamentales. Con esto, concluye, se está yendo en contra de la Constitución Política, y se terminaría en dado caso, ponderando un derecho fundamental como el derecho a la vida con la garantía de otro derecho fundamental como el de la sostenibilidad fiscal. Esta posibilidad plantea el germen de la derrota de los derechos fundamentales, como son los derechos sociales, frente a la sostenibilidad fiscal.

El Representante Dídier Burgos Ramírez expone que está bien que los colombianos tengan el derecho a la vivienda, el derecho a la salud, el derecho a la vida, a todos los derechos fundamentales, pero advierte que hay que tener cuidado de que al poner como principio fundamental la sostenibilidad fiscal, se nieguen estos derechos.

La Representante Alba Luz Pinilla Pedraza es crítica con el proyecto, pues implica colocar la sostenibilidad fiscal a la par con el derecho a la vida, y eso es violar los derechos fundamentales ya que se tendría que sostener que incluso la libertad del ser humano depende del crecimiento económico. Termina diciendo que las dotaciones básicas que dicen que 
la sostenibilidad fiscal va a regular, han sido hasta ahora, obligación del Estado Social de Derecho.

El Senador Juan Fernando Cristo Bustos, manifestó que no desea que este Proyecto de Reforma Constitucional limite la acción de los jueces.

\subsection{La sostenibilidad fiscal no afecta a los derechos sociales}

Son posiciones que niegan que a partir de la sostenibilidad fiscal pueda llegar a afectarse el Estado social de Derechos y los derechos fundamentales.

El Representante Guillermo Abel Rivera no es alarmista, le resta importancia a los alcances del proyecto y muestra un optimismo de no afectación a los derechos sociales por la sostenibilidad fiscal. Reconoce que el proyecto de acto legislativo busca de alguna manera poner en el mismo nivel la regla de estabilidad fiscal con los derechos fundamentales. Pero lo embarga la seguridad de que con ello no se podría ni siquiera igualar la importancia de una regla de estabilidad fiscal con un derecho fundamental, ya que es más que claro que predominaría el derecho fundamental. Es decir, un derecho fundamental en una colisión no podría verse afectado por la sostenibilidad fiscal, no sería vencido.

El Representante Alfredo Rafael Deluque, expone al Código Contencioso Administrativo, inclusive, como una herramienta muy valiosa para que esta sostenibilidad fiscal del Estado se dé de una manera adecuada. De tal suerte los sectores sociales de Colombia, pueden contar con una amplia gama de protección a sus derechos individuales y a sus derechos fundamentales.

El Representante Jaime Buenahora Febres Cordero comprende la sostenibilidad fiscal con la garantía de que el desarrollo de los derechos económicos y sociales sea una realidad en nuestro país, y como aquel principio que debe orientar la colaboración armónica de todas las ramas y órganos del poder público dentro de sus competencias. Procede a exponer las dudas que se tiene alrededor de este concepto y define que una de ellas es la referida a la elevación a rango constitucional del tema de sostenibilidad fiscal que aunque es aceptable, se cuestiona, ya que no se puede forzar el concepto para convertirlo en un derecho de todos y en un deber de las distintas ramas del Poder Público. Agrega a lo anterior que no es fácil encajar el concepto de sostenibilidad físcal como un derecho, ni haciendo referencia a los derechos fundamentales, ni de derechos sociales y económicos, ni tampoco de derechos colectivos, y 70 
menos suponer que es un deber del ciudadano. Concluye que más que ver como un derecho y deber al concepto de sostenibilidad fiscal, hay que entenderlo como un principio relacionado con la función administrativa.

E1 Senador Leonardo Barrera Montealegre habla sobre la sostenibilidad fiscal y la colaboración armónica, orientación a las ramas y órganos del Poder Público. Resalta que con la armonía que da la sostenibilidad, se pueden construir decisiones que favorezcan a todos los colombianos y no a unos privilegiados o a unos que se aprovechan de ciertas circunstancias del sistema.

\subsection{Evolución del proyecto en los ocho debates}

Desde el primer debate se evidencia la preocupación de los congresistas sobre el papel que tendrían los jueces en la realización de la sostenibilidad fiscal, puesto que, en ocasiones las decisiones judiciales implican gastos exorbitantes, los cuales no consultan la viabilidad económica del Estado.

Durante el segundo debate se discute sobre las decisiones de los jueces en las cuales siempre se tendrán un impacto fiscal; se modifica la condición de "derecho" a la sostenibilidad fiscal -como venía en el proyecto del Gobierno Nacional-, y en cambio, se eleva a rango de "principio", con el fin de que llegue a orientar a todas las ramas y órganos del poder público dentro de sus competencias, incluidos los jueces dentro de sus competencias.

En el tercer debate se considera necesario introducir una modificación al proyecto, en el sentido de que el principio de la sostenibilidad fiscal debía orientar la gestión de todas las Ramas y Órganos del Poder Público, mas no la colaboración armónica entre ellas. La anterior modificación tenía como propósito brindar una mayor protección al ciudadano en la realización de sus derechos económicos, sociales y culturales.

Para el cuarto debate, se muestra una preocupación frente a las decisiones de la rama judicial que ordenan gastos sin tener en cuenta la disponibilidad de recursos con los que cuenta el Gobierno.

En el quinto, se presentara una propuesta de adición en el sentido de que bajo ninguna circunstancia, se podrá invocar la aplicación del principio de sostenibilidad fiscal para menoscabar los derechos fundamentales. 
En el sexto debate, se muestra una inconformidad frente al contenido, teniendo en cuenta que dicha reforma constitucional estaba limitando el amparo de los derechos fundamentales, cuya realización y protección se encuentra a cargo de los jueces constitucionales.

En el séptimo debate se proponen modificaciones que vienen a resolver las discusiones sobre la sostenibilidad fiscal y su posible efecto negativo a las decisiones judiciales que protegen los derechos fundamentales. Estas son: (i) establecer la sostenibilidad fiscal como criterio y (ii) crear el incidente de impacto fiscal ante la Corte Constitucional en ciertos casos, con el fin de reflexionar sobre la forma como pueden cumplirse los fallos.

Y para finalizar en el octavo debate, el Senado realiza una modificación en el sentido de que el incidente fiscal pudiese interponerse ante las demás máximas corporaciones judiciales. Dicho texto fue aprobado por la Plenaria del Senado, cuyo texto fue acogido por la Comisión Accidental de Conciliación como el definitivo.

En suma de lo anterior es importante conocer cómo actúa la rama judicial y qué ha dicho el congreso respecto a este poder dentro del principio de sostenibilidad fiscal, en uno de los debates modificatorios del Acto legislativo, los congresistas deliberaron acerca de si era razonable consagrar la sostenibilidad fiscal como principio en la Constitución, el cual parecía limitar el trabajo de la Rama Judicial y su autonomía, ya que esta rama del poder público ordenaba gastos sin tener en cuenta la disponibilidad de recursos con los que contaba el Gobierno.

Respecto a esto, el congreso resolvió que el incidente de impacto fiscal pueda reflexionar, después de haber considerado los efectos fiscales, que puede lograr un cumplimiento efectivo dentro de un marco de sostenibilidad. Concluyendo, es importante reconocer que el acto legislativo se refiere a la sostenibilidad fiscal como el medio para orientar las ramas del poder público dentro de un marco de colaboración armónica. Teniendo en cuenta lo anterior, el Ministerio de Hacienda, tal como lo expresó en la Sentencia C-1052 de 2012, pretende que todas las ramas y órganos del poder público actúen y decidan en concordancia a la sostenibilidad fiscal, en pro de los objetivos del Estado Social de Derecho.

De esta manera, el diseño del Acto Legislativo 03 de 2011 responde al modelo de juez que según el Profesor Roa (2015) es neoconstitucionalista y garantista, pues es el que establece el poder judicial como el garante de 72 
los derechos constitucionales y adopta las medidas necesarias para cumplir con dicha función. De este modo, en principio no puede promoverse la regresividad de los derechos sociales con argumentos de sostenibilidad fiscal, pues no permitiría la orden de protección de los derechos a la disponibilidad efectiva de recursos por parte de la administración, y por el contrario, si se aplica el principio de progresividad, éste orientaría la aplicación de los derechos sociales, económicos y culturales.

\section{ANÁLISIS JURISPRUDENCIAL DE LA C-288 DE 2012}

En la sentencia mencionada, la Corte considera a la sostenibilidad fiscal como un instrumento para llegar a cumplir de manera progresiva la cláusula social del Estado de Derecho. Como instrumento permite un alcance material de los derechos constitucionales para todas las personas, de tal manera que se aseguren las condiciones necesarias para que genere el cumplimiento de las obligaciones prestacionales del Estado, bajo un criterio en el cual se consiga una mejor disciplina fiscal reduciendo así la discrecionalidad por parte de los Gobiernos a la hora de tomar decisiones que tengan que ver con la política económica, con la intención de crear una estabilidad macroeconómica y mejorar la estabilidad en las cuentas públicas.

No es correcto afirmar que al hablar de la garantía de la disponibilidad de los recursos se alude únicamente a la administración por parte de la rama ejecutiva del poder público. Más bien se puede considerar que al evolucionar las finanzas públicas se manifiesta que la sostenibilidad fiscal también depende de todos los involucrados en el nacimiento del gasto público -como pueden ser los jueces en sentencias estructurales o sobre derechos sociales-y a su vez al evolucionar el papel de los jueces se evidencia que la función que a ellos les corresponde como aplicadores del Derecho se ha transformado en la de garantes.

La sostenibilidad fiscal como criterio rector del poder público contribuye hacia el logro del equilibrio fiscal y la adecuada prestación de las funciones estatales, mediante el uso más racional de los recursos que ingresan a la economía para así armonizar el gasto y que los derechos que están limitados se puedan de esta manera cumplir permanentemente.

La sostenibilidad fiscal asume el papel de proteger el patrimonio público, para que cuando al principio de progresividad permita la complacencia de los derechos, otorgando prioridad a la asignación de gasto público para solucionar necesidades de salud, educación, 
saneamiento ambiental, agua potable, etc. Todo lo anterior, reconociendo la igualdad material y fomentando los derechos de las personas, pero sobre todo de aquellas que se encuentran menos favorecidas, en circunstancias de marginalidad o de debilidad manifiesta.

Es importante considerar que la progresividad anteriormente mencionada consiste en la obligación recaída sobre el estado de siempre ir hacia adelante con respecto a la consecución del goce pleno de garantías constitucionales; por lo tanto los Estados no pueden mantenerse inmóviles ante la satisfacción de dichas garantías, se debe por el contrario tomar medidas que impulsen el aumento de la cobertura de derechos sociales.

Además, la progresividad en el principio de sostenibilidad fiscal "implica la prohibición correlativa de la regresividad", es decir no se podría utilizar un argumento de sostenibilidad fiscal para dar paso atrás o aumentar las exigencias a la hora de efectivizar derechos fundamentales constitucionales.

La sostenibilidad fiscal se plantea como un principio de ámbito social pero que aspira a principio constitucional, tal y como se observa en la sentencia C 288 de 2012. Se resalta que:

La Corte encuentra que dicha estructura argumentativa [afectación de derechos] pierde fuerza de convicción cuando se advierte que la sostenibilidad fiscal, en realidad no es un principio constitucional, sino una herramienta para la consecución de los fines del Estado Social de Derecho. No es válido concluir, en ese orden de ideas, que la sostenibilidad fiscal redefina los objetivos esenciales del Estado, en tanto un instrumento de ese carácter no impone un mandato particular. Puede ser comprendida, a lo sumo, como una medida de racionalización de la actividad de las autoridades, pero en todo caso sometida a la consecución de los fines para el cual fue consagrada en la Constitución. Por ende, no es viable sostener que la sostenibilidad fiscal deba ponderarse con los principios constitucionales fundamentales, habida consideración que un marco o guía para la actuación estatal carece de la jerarquía normativa suficiente para desvirtuar la vigencia de dichos principios, limitar su alcance o negar su protección por parte de las ramas y órganos del Estado. En otros términos, no puede plantearse un conflicto normativo, ni menos aún una antinomia constitucional, entre la sostenibilidad fiscal y los 
principios fundamentales del Estado Social de Derecho, pues están en planos jerárquicos marcadamente diferenciados.

El instrumento por el cual se materializa para la rama judicial el principio de sostenibilidad fiscal es el incidente del impacto fiscal. En el momento en que al procurador general y a los ministros se les concede la facultad de presentar argumentos que exponen cómo en una sentencia judicial se afecta la sostenibilidad fiscal, ahí, la Corte interpreta el incidente del impacto fiscal. Para que este incidente tenga apertura, se debe proferir la sentencia por cualquiera de las máximas corporaciones judiciales, de esta manera se podrá solicitar la apertura del mismo, cuyo trámite será obligatorio.

Cabe recalcar que el incidente del impacto fiscal no habilita la modificación del fallo de la sentencia de manera obligatoria y rotunda, pero con este si se tiene el deber de considerar de una manera precisa los argumentos presentados por el procurador general y los ministros, para su cumplimiento. Se debe tener en cuenta que el incidente del impacto fiscal está delimitado por una serie de reglas, las cuales están determinadas según la etapa procesal en la que va el trámite.

Lo que se busca con el criterio del incidente del impacto fiscal es reforzar y limitar la toma de decisiones del gobierno en lo concerniente a las políticas económicas del país, considerando que mediante este procedimiento, constituido en dos etapas, se pueden presentar argumentos necesarios sobre posibles consecuencias del fallo de la sentencia que trascienda en el tema de finanzas públicas. Sólo si la Corte considera pertinente cambian los efectos del fallo - no su contenido-, se entrar a deliberar para poder llegar a una decisión equilibrada que incorpore, después de esta interlocución entre las altas cortes y los funcionarios mencionados, "un ámbito de protección de los derechos reconocidos judicialmente".

\section{CONCLUSIONES}

Teniendo en cuenta que el Acto Legislativo 03 de 2011 dispone que se debe "racionalizar la economía con el fin de conseguir en el plano nacional y territorial, en un marco de sostenibilidad fiscal, el mejoramiento de la calidad de vida de los habitantes, la distribución equitativa de las oportunidades y los beneficios del desarrollo", se entiende que ésta misma (sostenibilidad) es un instrumento que garantiza que la cláusula social del Estado de Derecho se pueda llegar a cumplir de manera progresiva. 
Hay pues una subordinación de la sostenibilidad fiscal al principio de progresividad, por lo que el deber que recae sobre el Estado de ir siempre hacia adelante en relación con el alcance del goce pleno de garantías constitucionales, es decir, que los derechos sociales sean garantizados de forma plena, no se desdibuja por la reforma constitucional de la sostenibilidad fiscal. La prioridad del gasto público social en el presupuesto nacional, se mantiene pese a la sostenibilidad.

Es importante reconocer que los debates presentados en la Corte Constitucional han avanzado de forma favorable hacia la sostenibilidad como el orientador armónico de las ramas y órganos, ya que es el que genera un equilibrio fiscal y una adecuada prestación de las funciones estatales que permiten armonizar el gasto público y cumplir los derechos sociales; y con apoyo en el Profesor Roa (2015), el juez neoconstitucional y garantista, es el que impulsa el principio de progresividad como mecanismo de racionalidad económica, supervive en este primer momento de análisis del Acto Legislativo 03 de 2011.

\section{REFERENCIAS BIBLIOGRÁFICAS}

Abramovich, V. \& Courtis, C. (2005). Los derechos sociales como derechos exigibles. Madrid: Trotta.

Colombia - Corte Constitucional de Colombia. Sentencia C 288 de 2012 (M. P. Luís Ernesto Vargas Silva: Abril 18 de 2012).

Colombia - Corte Constitucional de Colombia. Sentencia C 1052 de 2012 (M. P. Jorge Ignacio Pretelt Chaljub: Diciembre 5 de 2012).

Duarte, C., Frías, C., \& Ríos, M. (2014). Las garantías judiciales del procedimiento de provisión de viviendas dignas para los habitantes de zonas de alto riesgo a instancia de las acciones de tutela y popular. Revista de Derecho y Desarrollo, 2(3), 30-57. Recuperado de http://issuu.com/institutoomg/docs/redyd-2013-final

Ferrajoli, L. (2011). Poderes salvajes. La crisis de la democracia actual. Madrid: Trotta.

López, J., Bateman, A., Vengoechea, J. \& López, J. (2009). La garantía de los derechos sociales. Bogotá: Pontifica Universidad Javeriana y Editorial Ibañez. 
Ríos, M, (2015). La protección de los derechos subjetivos derivados de las facetas prestacional y de defensa del derecho a la vivienda digna en la jurisprudencia de tutela de la Corte Constitucional. Revista Temas Socio-Jurídicos, 33 (68), pp. 127-152.

Roa, J. (2015). El incidente de impacto fiscal y la legitimidad democrática de los tribunales constitucionales. Documentos de trabajo-Departamento de Derecho constitucional, 26, 5-27. Recuperado de http://icrp.uexternado.edu.co/wp-content/uploads/ 2015/05/DOC-DE-TRABAJO-26.pdf

Tushnet, M. (2013). Reflexiones sobre la protección judicial de los derechos sociales y económicos en el siglo XIX. En HENAO, J. (Ed.), Diálogos constitucionales de Colombia con el mundo (pp. 125-140). Bogotá: Universidad Externado de Colombia.

Upegui, J. (2009). Doce tesis en torno al concepto de Estado social de Derecho. Bogotá: Universidad Externado de Colombia 